\title{
Vortex-stream generation and enhanced propagation in a polariton superfluid
}

\author{
Giovanni Lerario, ${ }^{*}$ Anne Maître, Rajiv Boddeda $\odot$, Quentin Glorieux $\odot$, Elisabeth Giacobino, \\ Simon Pigeon ${ }^{\circledR}$, and Alberto Bramati ${ }^{\dagger}$ \\ Laboratoire Kastler Brossel, Sorbonne Université, CNRS, ENS, Université PSL, Collège de France, Paris 75005, France
}

(Received 30 July 2019; revised manuscript received 27 February 2020; accepted 4 March 2020; published 17 April 2020)

\begin{abstract}
In this work we implement an experimental configuration which exploits the specific properties of the optical bistability exhibited by the polariton system and we demonstrate the generation of a superfluid turbulent flow in the wake of a potential barrier. The propagation and direction of the turbulent flow are sustained by a support beam on distances an order of magnitude longer than previously reported. This technique is a powerful tool for the controlled generation and propagation of quantum turbulence and paves the way for the study of the hydrodynamics of quantum turbulence in driven-dissipative two-dimensional polariton systems.
\end{abstract}

DOI: 10.1103/PhysRevResearch.2.023049

\section{INTRODUCTION}

Exciton polaritons are bosonic quasiparticles arising from the strong coupling between excitons and photons. They have hybrid properties carried by their bare components and exhibit highly nonlinear behavior due to their excitonic content [1]. Since the first demonstration of Bose-Einstein condensation in exciton-polariton microcavities [2], these systems have become an attractive platform to study the hydrodynamics of quantum fluids. Due to the ability to prepare a polariton superfluid steady flow, the hydrodynamic nucleation of quantum turbulence was reported [3-6].

In the past decade, much effort has been made to control and manipulate the generation and propagation of vortices in polariton systems [7-10]. The vortices in a superfluid are collective excitations formed by a low-density core with quantized circulation around it, which makes them resilient to spontaneous decay, i.e., they are topologically protected. Since they are the fundamental block of the turbulence in superfluids, the study of their dynamics has attracted a great deal of interest.

Moreover, optical vortices are extremely promising for their application in quantum optical information science. In optics, modes carrying orbital angular momentum are used to encode and transport information [11]. Quantum vortices in planar microcavities are ideal candidates for binary encoding (due to their robustness to the perturbations and their clockwise or counterclockwise phase winding) towards on-chip technology. Furthermore, orbital angular momentum entanglement is at the forefront of quantum information science [12-17].

\footnotetext{
*giovanni.lerario@lkb.upmc.fr

†alberto.bramati@lkb.upmc.fr
}

Published by the American Physical Society under the terms of the Creative Commons Attribution 4.0 International license. Further distribution of this work must maintain attribution to the author(s) and the published article's title, journal citation, and DOI.
Different experimental configurations have been used to generate polariton vortices. In early experiments, quantized vortices have been observed in a condensate pumped with a continuous-wave (cw) nonresonant excitation [18], spontaneously emerging from the overlap between the condensate and a disordered potential landscape. Quantized vortices pairs also have been obtained using pulsed resonant pumping $[5,19]$. In this case, because of the dissipative nature of exciton polaritons, the vortex lifetime is limited by the polariton lifetime, thus limiting the study of their dynamics. With a cw resonant pumping, in order to ensure the absence of phase fixing and allow the appearance of topological excitations, many experiments were performed in the free propagation regime, outside the excitation area by the laser. However, also in this configuration, the polariton density and the propagation distance of the quantum fluid are limited by the polariton lifetime [6]. Thereafter, cw resonant and nonresonant pumping have been used to generate stationary vortices with in-plane engineering of the potential landscapes or even in optical parametric oscillation configurations [7-10]. However, all these cw configurations inhibit vortex free propagation-the vortex core is spatially pinned by the excitation intensity shape-and impose strong constraints on their free interaction.

A quasiresonantly driven semiconductor microcavity exhibits a bistable behavior when the pump laser is slightly blue detuned with respect to the polariton resonance [20]. The optical bistability, illustrated theoretically in Fig. 1(a), has been used in polariton systems for switching on and off the polariton population by triggering it with a weak control beam, allowing the achievement of polariton switches and logic gates [21,22]. Moreover, the upper branch of the bistability loop, where the polariton density and the nonlinear interactions are very strong, is closely linked to striking phenomena such as superfluidity and supersonic Cherenkov propagation [3].

In this work we consider the bistable hysteresis loop, highlighted in blue in Fig. 1(a), comprised between the lower and upper turning points of the bistability curve, where the system can be in either a linear or nonlinear regime. Remarkably, 


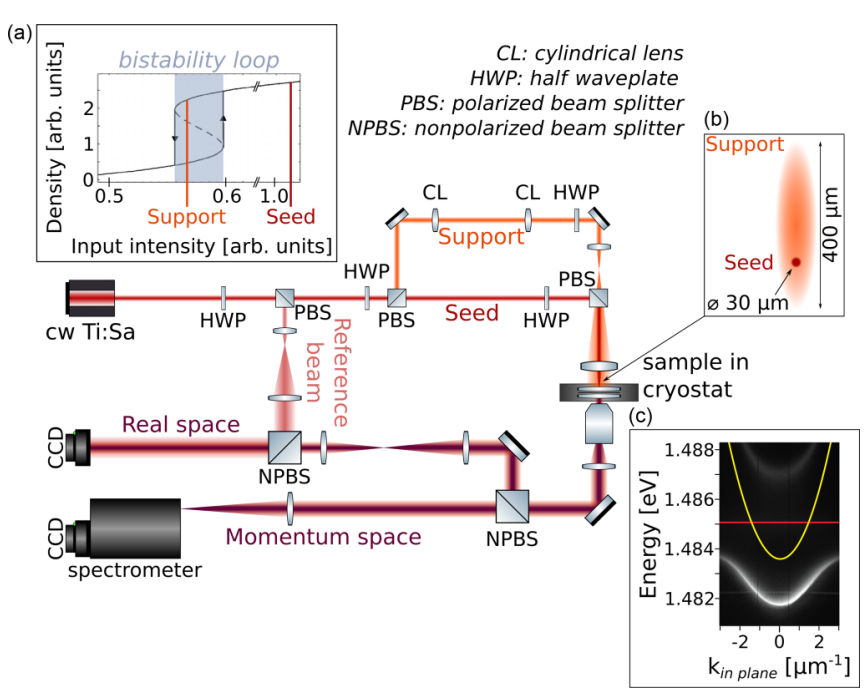

FIG. 1. Bistability, setup, and polariton dispersion. Inset (a) illustrates the positions of the support and seed beams with respect to the theoretical bistability curve. The support intensity ensures that the fluid stays inside the bistability loop, while the seed beam locks it on the upper branch. The highlighted region corresponds to the bistability loop, where the phase constraint imposed by the driving field is released. Also shown is a sketch of the optical setup. The excitation line consists of a support beam path (collimated on the sample surface) and a seed beam path (focused on the sample surface). The two beams are superposed at the sample surface. Using two cylindrical lenses in the excitation path, the support beam has an elliptic shape. Inset (b) shows the configuration of the seed and support on the sample surface. The detection line consists of two different paths projecting the real space and momentum space maps on two CCD cameras (see the text for further details). (c) Polariton dispersion under nonresonant excitation (2.54-eV cw laser). The strong coupling between the exciton transition (red line) and the photon mode (yellow line) gives rise to the upper and lower polariton branches.

as shown in a recent theoretical proposal [23], in this region the phase of the fluid is not fixed by the resonant driving field but is instead free to evolve and at the same time the polariton density can be high enough to observe spontaneous collective superfluid behavior mediated by the nonlinearity of the system.

We demonstrate that in the bistable regime, vortexantivortex pairs can be generated and controlled while their propagation is strongly enhanced far beyond the polariton free propagation length. To experimentally implement this configuration, we make use of a setup with two beams: a support beam on a large area with an intensity inside the bistability cycle and a localized seed beam with an intensity above the bistability regime that creates a superfluid flow and triggers the support into the upper branch of the bistability cycle [see Figs. 1(a) and 1(b)]. The support beam then sustains the superfluid propagation along the microcavity plane [see Fig. 1(b)]. Placing an obstacle in the supported region, we show that topological excitations can be hydrodynamically generated in the wake of the obstacle and propagate as long as the polariton superfluid remains in the bistable regime [23].
Furthermore, we show that the vortex propagation direction can be controlled by changing the in-plane wave vector of the support beam. Finally, modifying the support intensity, we can also control the density of vortices in the turbulent stream. The propagation distance appears to be only limited by the experimental need of a large homogeneous support beam, offering in principle limitless propagation of a quantum turbulent flow.

\section{RESULTS AND DISCUSSION}

The polariton microcavity under investigation is made of three $\mathrm{In}_{0.04} \mathrm{Ga}_{0.96} \mathrm{As} / \mathrm{GaAs}$ quantum wells embedded between two GaAs/AlAs-based distributed Bragg reflectors (DBRs), 21 pairs for the front DBR, and 24 pairs for the back one. The measurements are performed at cryogenic temperature and in transmission configuration.

The optical setup is sketched in Fig. 1. A cw single-mode Ti:sapphire laser at $831 \mathrm{~nm}$ pumps the polaritons in the quasiresonant excitation configuration. A first half waveplate (HWP) and polarizing beam splitter (PBS) extract a reference beam for the homodyne interferometric detection. The main beam is split into two different channels using a second HPW and PBS, one channel for the support beam and the other one for the seed beam. The seed beam is focused on the sample surface in an area with a FWHM of $30 \mu \mathrm{m}$ and intensity $\rho_{p}=10.6 \mathrm{~W} / \mathrm{mm}^{2}$. Making use of two cylindrical lenses along the support excitation line, the support beam intensity has an elliptical shape and it is collimated $\left(100 \times 400 \mu \mathrm{m}^{2}\right.$ FWHM) at the sample surface in order to cover a large area of the sample. The support input intensity is $5.8 \mathrm{~W} / \mathrm{mm}^{2}$. The two beams merge in the same excitation channel through a PBS, generating a seed spot focused near the edge of the large area illuminated by the support beam [Fig. 1(b)].

We can control the polariton group velocity, or flow velocity, by varying the angle of incidence $\theta$ of the pump with respect to the device plane $\left[k=k_{0} \sin (\theta)\right.$, with $k_{0}$ the wave vector of the pumping laser]. The detection channel is divided into two different paths by a beam splitter. The real space image of the sample surface is detected with a CCD camera in one arm, giving the map of the polariton fluid density, while a CCD camera placed in the second detection arm images the momentum space map. By using a spectrometer before the CCD camera in this second arm, the full dispersion curves can be measured by pumping the system with a nonresonant excitation (2.54-eV cw laser). Figure 1(c) shows the dispersion curves corresponding to the upper and lower polaritons arising from the strong coupling between the exciton level (red line) and the planar microcavity photon mode (yellow line). The cavity has a negative exciton-photon detuning $(-1.4 \mathrm{meV})$, the Rabi splitting is $5.2 \mathrm{meV}$, and the polariton lifetime is 14 ps. The experiments are operated close to resonance with the lower polariton mode.

Figures 2(a) and 2(b) show the seed-only and supportonly momentum space maps in the quasiresonant excitation configuration, respectively. Both excitation beams have the same wave vector $\left(\left|k_{y}\right|=0.6 \mu \mathrm{m}^{-1}\right.$ and $\left.\left|k_{x}\right|=0 \mu \mathrm{m}^{-1}\right)$. The flow speed depends on the polariton dispersion according to the formula $v_{\text {flow }}=\frac{1}{\hbar} \frac{\partial E}{\partial k}$. In our case, the flow speed is 

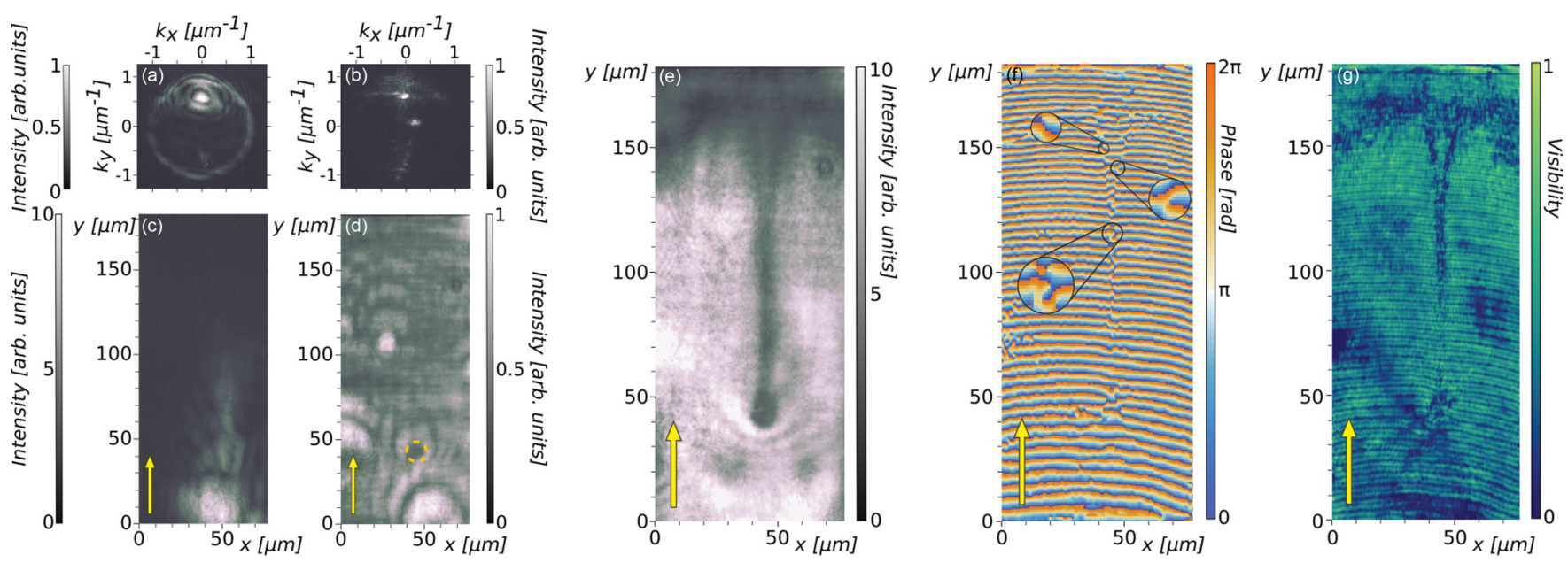

FIG. 2. Vortex generation and propagation. (a) Seed-only and (b) support only momentum maps. The excitation detuning is 0.26 meV for both beams. (c) Seed-only and (d) support-only space intensity distributions. The arrows indicate the flow direction. The position of the obstacle in (d) is shown by the yellow dashed circle. Note that for the support only the intensity scale is divided by a factor of 10 . Also shown are (e) intensity, (f) phase, and (g) visibility maps when the support and the seed simultaneously excite the sample. Due to the presence of the support beam, the polariton population is drastically increased in a wide area. The vortices flowing in the wake of the defect generate a long dark shadow in the fluid propagation direction. The time integration over a continuous flow of vortices induces a blurring of the phase [bottom close-up in (f)] along the vortex stream and a consequent decrease of the visibility. Fork-shaped patterns [middle close-up in (f)] demonstrate the formation of localized vortices and the top close-up in (f) indicates the formation of unstable gray solitons at the border of the locked area (see the text for further details). Panels (e)-(g) are plotted keeping fixed the real space image orientation.

$0.9 \mu \mathrm{m} / \mathrm{ps}$ with the flow direction along the support beam major axis.

The excitation energy is close to resonance with the lower polariton branch at low excitation power. The scattering ring in Fig. 2(a) $\left(|k|=1 \mu \mathrm{m}^{-1}\right.$ radius) highlights the detuning of the excitation with respect to the lower polariton resonance, which is $0.26 \mathrm{meV}$ at the excitation in-plane wave vector. Using a small excitation spot in the momentum space and increasing the excitation power, the blueshift of the polariton dispersion induced by the polariton-polariton repulsive interactions allows for the abrupt increase of the polariton density when the system locks to the excitation laser energy. With a blue-detuned driving field, one can then observe a bistability cycle $[20,23]$ as for any Kerr medium in a microresonator.

In the case of support only [Fig. 2(d)], the system lies in the lower part of the bistability loop [note that in Fig. 2(d), the support-only intensity map, the scale is divided by a factor of 10 with respect to the other intensity maps] and few photons enter the microcavity. The polariton flow propagation in the disordered random potential of the microcavity leads to interference effects offering bright and dark spots. One dark spot, indicated by the orange dashed circle in Fig. 2(d), is noticeable by its size, approximately $8 \mu \mathrm{m}$ in diameter, and corresponds to a high potential barrier, which will create vortices downstream.

When the seed beam is superposed with the support beam [Fig. 2(e)], the local increase of the polariton-polariton interactions blueshifts the mode resonance, allowing the jump of the system to the upper part of the bistability loop, together with a drastic increase of the polariton density. The high polariton density region extends over a much wider area than where the seed beam is located and it is only limited by the support size. This is possible because the intensity of the support beam is carefully adjusted to stay within the hysteresis loop of the bistability curve.

The sound velocity depends on the polariton density, $c_{\text {sound }}=\sqrt{\frac{\hbar g|\psi|^{2}}{m_{\mathrm{pol}}}}$, with $g$ the polariton-polariton interaction constant, $m_{\mathrm{pol}}$ the polariton mass, and $\psi$ the polariton wave function. The $c_{\text {sound }}$ is $0.78 \mu \mathrm{m} / \mathrm{ps}$ in the area where the support beams locks to the upper part of the bistability cycle. The flow velocity $(0.9 \mu \mathrm{m} / \mathrm{ps}$, as mentioned above $)$ is then larger than the speed of sound and the propagation is supersonic, allowing for the generation of Cherenkov wavefronts upstream the obstacle and for the propagation of perturbations downstream. Here a long dark shadow appears in the wake of the defect along the support propagation direction. The presence of a potential barrier on the polariton flow leads to the generation of vortex-antivortex pairs. The time integration over a flowing stream of vortex pairs continuously generated at the defect position leads to a shadow in the wake of the defect, as predicted in Ref. [23] and reported here. Because of the time integration, the intensity of the shadow in the wake of the defect depends on the vortex generation rate: The higher the generation rate, the darker the shadow. According to the theoretical predictions, the vortex generation rate is maximal close to the lower limit of the bistability loop, which allows us to maximize the contrast and to clearly observe the turbulence stream. The vortices propagate for more than $120 \mu \mathrm{m}$, an order of magnitude longer than the distance supported by the polariton lifetime, that is, $12 \mu \mathrm{m}$. This propagation distance is only limited by the support field profile, which needs to be as flat as possible.

To further justify that the observed shadow indeed results from the time averaging of a quantum turbulent flow, the phase pattern of the fluid is analyzed. A homodyne interferometer is used to probe the phase pattern associated with Fig. 2(e). A 


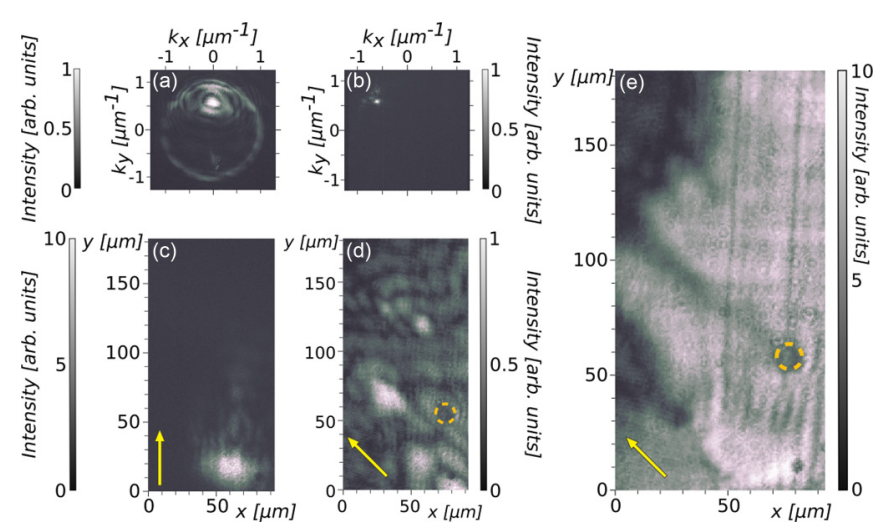

FIG. 3. Control of the vortex propagation direction. (a) Seedonly and (b) support-only momentum maps. The excitation detuning is $0.26 \mathrm{meV}$ for both beams, but they are at an angle of about $40^{\circ}$ with respect to each other. The real space images of the (c) seed-only and (d) support-only intensity show the two different propagation directions. The arrows indicate the flow direction. Note that the support-only intensity scale is divided by a factor of 10. (e) Intensity map when the support and seed simultaneously excite the sample. The vortex stream flows in the direction fixed by the support beam and propagates for $90 \mu \mathrm{m}$.

portion of the support beam is extracted along the excitation line and used as a reference beam with a homogeneous phase, making it interfere on the CCD camera with the signal coming from the microcavity. The time integration over a continuous flow of the vortex stream results in the blurring of the phase in the wake of the defect. Indeed, the bottom inset of the interferogram [Fig. 2(f)] presents such a blurred phase at a position coinciding with the shadow stream. To explain this phenomenon we report in Fig. 2(g) the corresponding visibility which decreases along the turbulent streamline. Fork-shaped patterns associated with localized vortices along the flow are also visible in the central zoom in Fig. 2(f). At the border of the locked area the polariton density is decreasing because of the Gaussian shape of the support beam. Therefore, in this region the vortex core radius increases due to the increase of the healing length. This effect induces the merging of the ejected vortices and the formation of unstable gray solitons [top close-up in Fig. 2(f)], which repel each other and generate the $Y$ shape observed in the experiment. This $Y$ shape has also been observed for solitons in previous experiments [6].

Moreover, the seed-support configuration allows us to control the vortex propagation direction via the support wavevector orientation. Figure 3(e) shows the stream of vortices flowing with a nonzero component in $k_{x}$. Here the seed momentum is $\left|k_{y}\right|=0.6 \mu \mathrm{m}^{-1}$ [and $\left|k_{x}\right|=0 \mu \mathrm{m}^{-1}$, Fig. 3(a)], while the support momentum orientation, with the same module, is displaced at about $40^{\circ}$ with respect to the seed one [Fig. 3(b)]. Figures 3(c) and 3(d) show the seed-only and support-only space maps, respectively. As evident from Figs. 2 and 3, the vortices stream flows with a propagation direction defined by the support beam direction.

As specified above, the intensity of the shadow depends on the vortex generation rate in time-integrated measurements. By increasing the support density above the bistable regime the vortex generation is strongly inhibited because the fluid
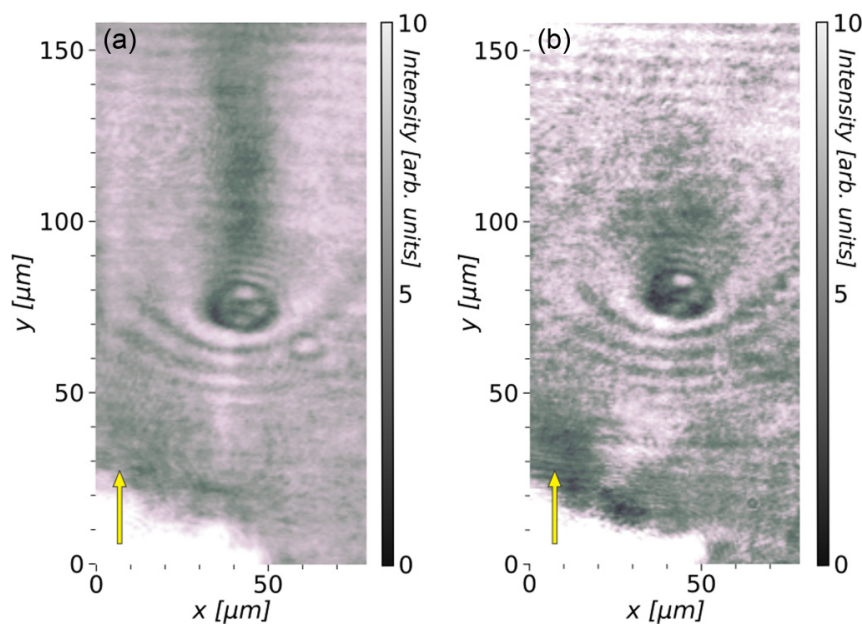

FIG. 4. Vortex suppression. (a) Vortex-stream generation and propagation in the wake of the defect for a $5.8-\mathrm{W} / \mathrm{mm}^{2}$ support power density. (b) Increasing the support intensity up to $6 \mathrm{~W} / \mathrm{mm}^{2}$, the phase of the support is strongly imprinted on the fluid and, consequently, the vortex generation is inhibited.

phase is fixed to the support beam phase. Experimentally, this translates into the suppression of the shadow in the wake of the defect when increasing the support power as predicted in Ref. [23]. In Fig. 4(a) we can observe the vortex stream propagating in the wake of the defect; here the experimental configuration and parameters are identical to the ones previously described. When the excitation density is increased to $6 \mathrm{~W} / \mathrm{mm}^{2}$ [Fig. 4(b)], which is just above the limit of the bistable regime, the vortex stream is completely suppressed by the phase constraint imposed by the support beam, with almost no change in the fluid properties (speed and density).

\section{CONCLUSION}

We have demonstrated the generation of a vortex stream flowing over more than $120 \mu \mathrm{m}$ in the plane of a polariton microcavity, implementing an original method proposed by Pigeon and Bramati [23]. In this approach we used the bistability to release the phase fixing of the polariton fluid to the resonant support beam while maintaining the particle density sufficiently high to observe topological excitations. Vortices could then be sustained without being limited by the polariton lifetime and could flow over macroscopic distances. Additionally, we demonstrated that the vortex-stream direction can be controlled by the orientation of the support beam wave vector and the vortex density can be tuned by varying the support intensity within the bistability cycle. This implementation of an experimental configuration allows for the controlled propagation and interaction of polariton vortices, paving the way for the on-chip macroscopic propagation and efficient manipulation of topological excitations. Our results are a significant breakthrough towards the study of quantum turbulence hydrodynamics in two-dimensional quantum fluids of light. The main advantage of the technique we have developed is given by the possibility to study quantum turbulence in a stationary regime, thus avoiding the need of a very high 
temporal resolution (in the picosecond range) due to the short polariton lifetime. As an example, this configuration could be used for the study of the energy cascade in two-dimensional exciton polariton systems and for the identification of the superfluid Reynolds number as proposed in [24,25]. These experiments have been inaccessible in the polaritons.

\section{ACKNOWLEDGMENTS}

This work received funding from French ANR grants (C-FLigHT 138678 and QFL) and from the European Union Horizon 2020 research and innovation program under Grant Agreement No. 820392 (PhoQuS). Q.G. and A.B. thank the Institut Universitaire de France for support.
[1] M. Vladimirova, S. Cronenberger, D. Scalbert, K. V. Kavokin, A. Miard, A. Lemaître, J. Bloch, D. Solnyshkov, G. Malpuech, and A. V. Kavokin, Polariton-polariton interaction constants in microcavities, Phys. Rev. B 82, 075301 (2010).

[2] J. Kasprzak, M. Richard, S. Kundermann, A. Baas, P. Jeambrun, J. M. J. Keeling, F. M. Marchetti, M. H. Szymańska, R. André, J. L. Staehli, V. Savona, P. B. Littlewood, B. Deveaud, and L. S. Dang, Bose-Einstein condensation of exciton polaritons, Nature (London) 443, 409 (2006).

[3] A. Amo, J. Lefrère, S. Pigeon, C. Adrados, C. Ciuti, I. Carusotto, R. Houdré, E. Giacobino, and A. Bramati, Superfluidity of polaritons in semiconductor microcavities, Nat. Phys. 5, 805 (2009).

[4] G. Roumpos, M. D. Fraser, A. Löffler, S. Höfling, A. Forchel, and Y. Yamamoto, Single vortex-antivortex pair in an excitonpolariton condensate, Nat. Phys. 7, 129 (2011).

[5] G. Nardin, G. Grosso, Y. Léger, B. Pietka, F. Morier-Genoud, and B. Deveaud-Plédran, Hydrodynamic nucleation of quantized vortex pairs in a polariton quantum fluid, Nat. Phys. 7, 635 (2011).

[6] A. Amo, S. Pigeon, D. Sanvitto, V. G. Sala, R. Hivet, I. Carusotto, F. Pisanello, G. Lemenager, R. Houdre, E. Giacobino, C. Ciuti, and A. Bramati, Polariton superfluids reveal quantum hydrodynamic solitons, Science 332, 1167 (2011).

[7] D. Sanvitto, S. Pigeon, A. Amo, D. Ballarini, M. De Giorgi, I. Carusotto, R. Hivet, F. Pisanello, V. G. Sala, P. S. S. Guimaraes, R. Houdré, E. Giacobino, C. Ciuti, A. Bramati, and G. Gigli, All-optical control of the quantum flow of a polariton condensate, Nat. Photon. 5, 610 (2011).

[8] R. Dall, M. D. Fraser, A. S. Desyatnikov, G. Li, S. Brodbeck, M. Kamp, C. Schneider, S. Höfling, and E. A. Ostrovskaya, Creation of Orbital Angular Momentum States with Chiral Polaritonic Lenses, Phys. Rev. Lett. 113, 200404 (2014).

[9] T. Boulier, E. Cancellieri, N. D. Sangouard, Q. Glorieux, A. V. Kavokin, D. M. Whittaker, E. Giacobino, and A. Bramati, Injection of Orbital Angular Momentum and Storage of Quantized Vortices in Polariton Superfluids, Phys. Rev. Lett. 116, 116402 (2016).

[10] R. Hivet, E. Cancellieri, T. Boulier, D. Ballarini, D. Sanvitto, F. M. Marchetti, M. H. Szymanska, C. Ciuti, E. Giacobino, and A. Bramati, Interaction-shaped vortex-antivortex lattices in polariton fluids, Phys. Rev. B 89, 134501 (2014).

[11] A. E. Willner, H. Huang, Y. Yan, Y. Ren, N. Ahmed, G. Xie, C. Bao, L. Li, Y. Cao, Z. Zhao, J. Wang, M. P. J. Lavery, M. Tur, S. Ramachandran, A. F. Molisch, N. Ashrafi, and S. Ashrafi, Optical communications using orbital angular momentum beams, Adv. Opt. Photon. 7, 66 (2015).
[12] A. Mair, A. Vaziri, G. Weihs, and A. Zeilinger, Entanglement of the orbital angular momentum states of photons, Nature (London) 412, 313 (2001).

[13] B. C. Hiesmayr, M. J. De Dood, and W. Löffler, Observation of Four-Photon Orbital Angular Momentum Entanglement, Phys. Rev. Lett. 116, 073601 (2016).

[14] R. Fickler, G. Campbell, B. Buchler, P. K. Lam, and A. Zeilinger, Quantum entanglement of angular momentum states with quantum numbers up to 10,010, Proc. Natl. Acad. Sci. USA 113, 13642 (2016).

[15] E. Nagali, L. Sansoni, F. Sciarrino, F. De Martini, L. Marrucci, B. Piccirillo, E. Karimi, and E. Santamato, Optimal quantum cloning of orbital angular momentum photon qubits through Hong-Ou-Mandel coalescence, Nat. Photon. 3, 720 (2009).

[16] D. Giovannini, E. Nagali, L. Marrucci, and F. Sciarrino, Resilience of orbital-angular-momentum photonic qubits and effects on hybrid entanglement, Phys. Rev. A 83, 042338 (2011).

[17] L. Marrucci, E. Karimi, S. Slussarenko, B. Piccirillo, E. Santamato, E. Nagali, and F. Sciarrino, Spin-to-orbital conversion of the angular momentum of light and its classical and quantum applications, J. Opt. 13, 064001 (2011).

[18] K. G. Lagoudakis, M. Wouters, M. Richard, A. Baas, I. Carusotto, R. André, L. S. Dang, and B. Deveaud-Plédran, Quantized vortices in an exciton-polariton condensate, Nat. Phys. 4, 706 (2008).

[19] G. Lerario, A. Fieramosca, F. Barachati, D. Ballarini, K. S. Daskalakis, L. Dominici, M. De Giorgi, S. A. Maier, G. Gigli, S. Kéna-Cohen, and D. Sanvitto, Room-temperature superfluidity in a polariton condensate, Nat. Phys. 13, 837 (2017).

[20] A. Baas, J. P. Karr, H. Eleuch, and E. Giacobino, Optical bistability in semiconductor microcavities, Phys. Rev. A 69, 023809 (2004).

[21] M. De Giorgi, D. Ballarini, E. Cancellieri, F. M. Marchetti, M. H. Szymanska, C. Tejedor, R. Cingolani, E. Giacobino, A. Bramati, G. Gigli, and D. Sanvitto, Control and Ultrafast Dynamics of a Two-Fluid Polariton Switch, Phys. Rev. Lett. 109, 266407 (2012).

[22] D. Ballarini, M. De Giorgi, E. Cancellieri, R. Houdré, E. Giacobino, R. Cingolani, A. Bramati, G. Gigli, and D. Sanvitto, All-optical polariton transistor, Nat. Commun. 4, 1778 (2013).

[23] S. Pigeon and A. Bramati, Sustained propagation and control of topological excitations in polariton superfluid, New J. Phys. 19, 095004 (2017).

[24] M. T. Reeves, T. P. Billam, B. P. Anderson, and A. S. Bradley, Inverse Energy Cascade in Forced Two-Dimensional Quantum Turbulence, Phys. Rev. Lett. 110, 104501 (2013).

[25] M. T. Reeves, T. P. Billam, B. P. Anderson, and A. S. Bradley, Identifying a Superfluid Reynolds Number via Dynamical Similarity, Phys. Rev. Lett. 114, 155302 (2015). 